\title{
EFECTIVIDAD DE LA AZADIRACHTINA EN LA INHIBICIÓN DE LA EMBRIOGÉNESIS DE AEGORHINUS SUPERCILIOSUS (GUÉRIN) (COLEOPTERA: CURCULIONIDAE)
}

\author{
AZADIRACHTIN EFFECTIVITY IN EMBRYOGENESIS INHIBITION OF \\ AEGORHINUS SUPERCILIOSUS (GUÉRIN) (COLEOPTERA: CURCULIONIDAE)
}

\author{
Alfonso Aguilera P.3.; Mario Zampezzi V. ${ }^{1,2}$; Ximena Araneda D. ${ }^{1}$; \\ Carlos Klein K. ${ }^{4}$; Ramón Rebolledo . $^{3}$
}

\section{RESUMEN}

La frambuesa es una fruta de exportación, cuyo cultivo en el sur de Chile es afectado seriamente por el curculiónido Aegorhinus superciliosus (Guérin) o cabrito del frambueso (CF), insecto nativo de Chile.

La biología del CF es poco conocida y también su control. Se ha intentado reducir al adulto con insecticidas convencionales; sin embargo, en la práctica se dificulta su uso por coincidir la presencia del insecto adulto con periodos de floración y cosecha de fruta. Recientemente se están incorporando hongos entomopatógenos, pero aún no se aplican masivamente.

En este trabajo se experimentó con azadirachtina, utilizando el producto comercial Neem como regulador de crecimiento. Bajo condiciones de laboratorio se aplicaron seis dosis de Neem: $1 \mathrm{ml} ; 2 \mathrm{ml} ; 3 \mathrm{ml} ; 4 \mathrm{ml} ; 5 \mathrm{ml} ; 6 \mathrm{ml} \mathrm{de} \mathrm{Neem} \mathrm{por} \mathrm{litro} \mathrm{de} \mathrm{agua.} \mathrm{Además,}$ de un testigo sin insecticida.

Los adultos del CF se mantuvieron confinados con follaje de frambueso tratados con Neem durante cinco días y en postura por treinta días. Durante este periodo se determinó: cantidad de huevos, cantidad de larvas emergidas, porcentaje de larvas nacidas, grado de eficacia, dosis media efectiva (DE50) y dosis noventa efectiva (DE90).

Los tratamientos con Neem fueron significativamente diferentes al testigo, presentando menor postura, menor emergencia de larvas y mayor grado de efectividad. Con $2 \mathrm{ml}$ de Neem se obtuvo la menor postura; sin embargo, la dosis de $5 \mathrm{ml}$ de Neem presentó la menor cantidad de larvas emergidas y el menor porcentaje de emergencia de larvas. La DE50 se determinó en 0,0290 ml de Neem y la DE90 en $8,923 \mathrm{ml}$ de Neem por litro de agua.

Con los antecedentes expuestos se concluyó que el Neem tiene un efecto que altera la postura y la embriogénesis del CF.

Palabras clave: Frambuesas, Aegorhinus superciliosus, azadirachtina, Curculionidae, Chile.

\begin{abstract}
Raspberry is cultivated in the southern part of Chile mainly for export. Its fruit is severely affected by the raspberry weevil (RW) Aegorhinus superciliosus (Guérin), a Curculionidae native of Chile.

Little is known about the biology RW and control measures. Conventional insecticides have been tried against the adult, however, their use has been hindered in the practice by the coincidence of the presence of the mature insect with flowering and fructification periods. Entomopathogenic fungi are being incorporated lately, but still not massively used.

This work aimed to evaluate azadirachtin, using the commercial product Neem as a growth regulator. Six doses of Neem, 0.5, 1, 2, 3, 4, and $5 \mathrm{ml} / \mathrm{L}$ of water, were applied under laboratory conditions, maintaining a check with no insecticide. $R W$ adults stayed confined on the foliage of raspberry bushes treated with Neem during five days and in thirty days during egglaying. Number of eggs, number of emerged larva, percentage of born larva, effectiveness; effective half dose (DE50) and ninety effective dose (DE90) were determined.

Neem treatments had significantly lower egg-laying amount, lower emergence of larvae and greater effectiveness, compared to the untreated check. The lowest egg-laying number was obtained with $2 \mathrm{ml}$ of Neem, however, $5 \mathrm{ml}$ of Neem resulted in the smallest number and percent of emerged larvae. DE50 and DE90 were found to be 0.0290 and $8.923 \mathrm{ml}$ of Neem for liter of water, respectively. It was concluded that Neem can significantly alter egg-laying and embryogenesis of the RW.
\end{abstract}

Key words: Raspberry, Aegorhinus superciliosus, azadirachtin, Curculionidae, Chile.

\footnotetext{
1 Universidad Católica de Temuco. Temuco, Chile.

2 Centro Regional de Investigación INIA Carillanca. Temuco

3 Universidad de La Frontera. Temuco, Chile. E-mail: aaguiler@ufro.cl

4 Parcela El Tirol. Maipo, Temuco, Chile.
}

Fecha de Recepción: 18 Mayo 2007

Fecha de Aceptación: 03 Octubre 2007 


\section{INTRODUCCIÓN}

La frambuesa, Rubus idaeus L., en la actualidad es uno de los berries importantes en Chile, con distribución desde la Región de Coquimbo a la Región de Aysén.

Este rubro constituye una alternativa frutal interesante para la zona sur del país y pasó a constituir un nuevo agroecosistema, proporcionando un hábitat favorable para numerosas especies de insectos nativos (Aguilera 1994, 1995; González, 1985), siendo el cabrito del frambueso (CF), Aegorhinus superciliosus (Guérin) (Coleoptera: Curculionidae), una de las principales y más importantes plagas que dañan los cultivos de berries en la zona sur de Chile. El daño más severo en frambueso es causado por el estado larvario, de hábito subterráneo, atacando la raíz principal donde construye galerías destruyendo los haces conductores del sistema radical (Guerrero \& Aguilera, 1989).

En la zona sur de Chile se han incorporado varios berries como productos exportables del país; sin embargo, dentro de éstos la frambuesa se ha transformado en un rubro con grandes dificultades de exportación, debido a problemas asociados a los insectos y pesticidas (González, 1985).

Se estima que el control de plagas en arbustos frutales deberá lograrse mediante el diseño de una estrategia que armonice la fenología del cultivo, el manejo del huerto, destino de la producción y el conocimiento de la presencia de biorreguladores o poblaciones de depredadores benéficos que actúen como control natural sobre estas plagas (Neira \& Mundaca, 1982).

Guerrero et al. (1999) determinaron el hongo Metarhizium anisopliae var. anisopliae asociado con Hylamorpha elegans, Phytoloema hermanni, Schizochelus breviventris y Brachysternus prasinus (Coleoptera: Scarabaeidae) y con Aegorhinus sp. y Graphognatus leucoloma (Coleoptera: Curculionidae). Entre los hongos encontrados con mayor frecuencia destacan Beauveria y Metarhizium, los cuales se utilizan con gran éxito en el control de plagas en el mundo. A través de evaluaciones experimentales de los hongos colectados se han seleccionado cepas para combatir importantes insectos plagas de la zona centro-sur y sur de Chile (Gerding et al., 2002). También se mencionan nematodos del género Steinernema y Heterorhabditis y a Centistes sp, un himenóptero bracónido (Prado, 1991), al grillo rojo, larvas y adultos de carábidos, larvas de asílidos (Devotto \& Gerding, 2001).
Según Rodríguez et al. (2005), las larvas de las especies de curculiónidos, tales como el CF, capachito de los frutales y gorgojo de la frutilla, consumen raíces y raicillas en gran número de cultivos, destacándose los frutales menores, los que han mostrado un alto grado de susceptibilidad, al estar expuestos a aislamientos específicos de Metarhizium anisopliae.

Respecto a la regulación poblacional artificial del CF con insecticidas convencionales orgánicos de síntesis (González \& Varas, 1989) se han efectuado estudios para el control del estado larvario; sin embargo, la efectividad no ha sido del todo exitosa debido al hábito de vida de la larva, que por lo demás representa más del $80 \%$ del total del periodo de vida del insecto (Aguilera \& Rebolledo, 2001). También se ha intentado el control del adulto con insecticidas orgánicos de síntesis de largo efecto residual, con un éxito relativo. El mayor problema de estos productos son su alta toxicidad, larga carencia y persistencia, lo que limita su aplicación en periodos de cosecha, precisamente cuando se produce la mayor emergencia de adultos en el cultivo (Guerrero \& Aguilera, 1989). También se han efectuado experiencias con insecticidas orgánicos sintéticos conocidos como reguladores del crecimiento que inhiben la formación de quitina (Soler, 1994; Aguilera, 1995).

Considerando la propiedad de regulador del crecimiento de la azadirachtina expresada en los trabajos de Steets \& Schmutterer (1975); Steets (1976); Rembold \& Sieber (1981); Ascher (1981); Schulz (1981); Ascher et al. (1984); Heyde et al. (1984); Ascher et al. (1986); Schmidt \& Pesel (1986); Schmutterer (1987) en diferentes órdenes de insectos como Orthoptera, Lepidoptera, Coleoptera, Hemiptera, Diptera e Hymenoptera, y por constituir un insecticida botánico natural, no contaminante del medio ambiente, no tóxico para el hombre y de acuerdo al hábito alimentario del adulto del $\mathrm{CF}$, este trabajo tuvo como objetivo determinar la efectividad de la azadirachtina como regulador de crecimiento en el periodo de oviposición del CF para inhibir la embriogénesis.

\section{MATERIALES Y MÉTODOS}

El estudio se realizó entre noviembre 2005 y febrero 2006 en el laboratorio de Entomología del Centro Regional de Investigación INIA Carillanca, 
ubicado a $38^{\circ} 41^{\prime}$ latitud sur y $72^{\circ} 25^{\prime}$ longitud oeste, en el kilómetro 10 del camino Cajón a Vilcún.

Para evaluar el efecto de la azadirachtina en la embriogénesis del CF, a partir de noviembre 2005 se colectaron 480 ejemplares adultos del insecto en un huerto de frambueso ubicado en la comuna de Vilcún, a un costado de la carretera en el kilómetro 12 que une al pueblo de Cajón con Vilcún. El material colectado en terreno se colocó en envases de plástico de cinco litros. En el laboratorio este material se traspasó a envases plásticos de veinte litros, colocados sobre mesones de madera, recibiendo alimentación diaria de brotes frescos de frambueso.

En el laboratorio se registró a diario la temperatura, con un termómetro de máxima y mínima, y el registro diario de la humedad relativa con un higrómetro de pelo. La temperatura durante el ensayo correspondió a una mínima media de 15,7 ${ }^{\circ} \mathrm{C}$ y una máxima media de $27,5^{\circ} \mathrm{C}$. La humedad relativa fluctuó entre un mínimo de $57,5 \%$ y un máximo de $63,5 \%$. El promedio de la temperatura durante el ensayo en laboratorio fue de $21,6^{\circ} \mathrm{C}$ y el promedio de humedad relativa fue de $60,5 \%$, con un fotoperiodo natural aproximado de 16 horas luz y 8 horas de oscuridad.

Para realizar el ensayo se ocuparon frascos de conservas de $1.000 \mathrm{cc}$, placas Petri, vasos de precipitado, pipetas, pHmetro, aspersores, papel filtro, agua destilada, papel absorbente, pinzas, pinceles de pelo fino, marcadores. Se prepararon cinco dosis de azadirachtina, en función del producto comercial Neem: 0,5 ml/l de agua (T1), $1 \mathrm{ml} / \mathrm{l}$ de agua (T2), $2 \mathrm{ml} / \mathrm{l}$ de agua (T3), $4 \mathrm{ml} / \mathrm{l}$ de agua (T4), $5 \mathrm{ml} / \mathrm{de}$ agua (T5). Cada dosis de Neem se aplicó sobre hojas frescas de frambueso durante cinco días consecutivos, renovando diariamente el alimento a los ejemplares del CF. También se dispuso de un tratamiento testigo (T0) sin aplicación de Neem. El pH del agua utilizada para las preparaciones y aspersiones en las dosis aplicadas en este ensayo tuvo una variación de 5,2 a 5,3, considerándose este $\mathrm{pH}$ adecuado para las exigencias del Neem, de acuerdo a las instrucciones de la etiqueta del producto.

Después de cinco días, los ejemplares adultos del CF (Figura 1), alimentados con brotes de frambuesas frescos con azadirachtina se mantuvieron durante 30 días bajo control, alimentándolos con brotes de frambuesas libres de insecticida y registrándose en ambos periodos la oviposición diaria de cada unidad experimental, que consistió en un disco Petri de $90 \mathrm{~mm}$ con cinco ejemplares de CF adultos (Figura 3). Los huevos se separaron diariamente y se trasladaron a discos Petri de 30 $\mathrm{mm}$ con fondo de papel filtro humedecido con agua destilada. Para ello se ocupó un aspersor manual de 1 litro. Los huevos (Figura 2) se mantuvieron

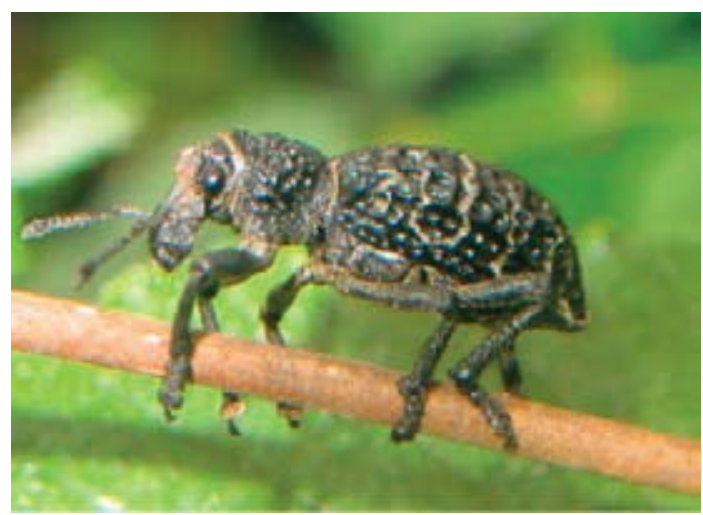

Figura 1. Adulto, Aegorhinus superciliosus.

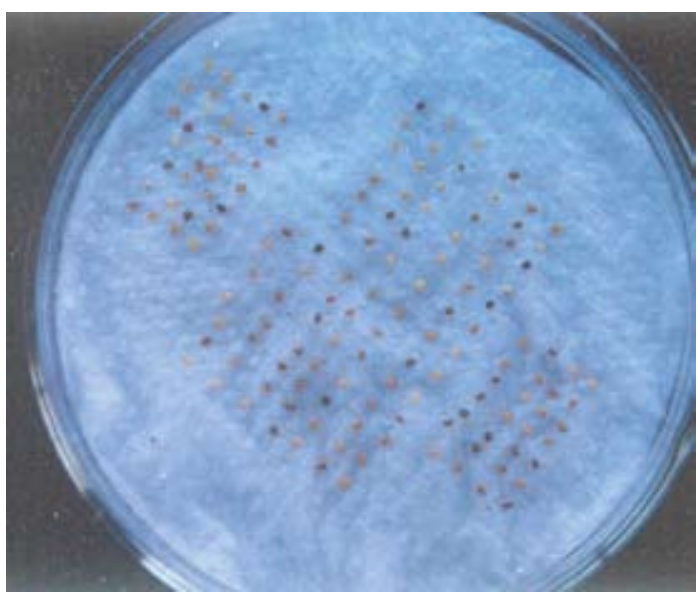

Figura 2. Huevos, Aegorhinus superciliosus.

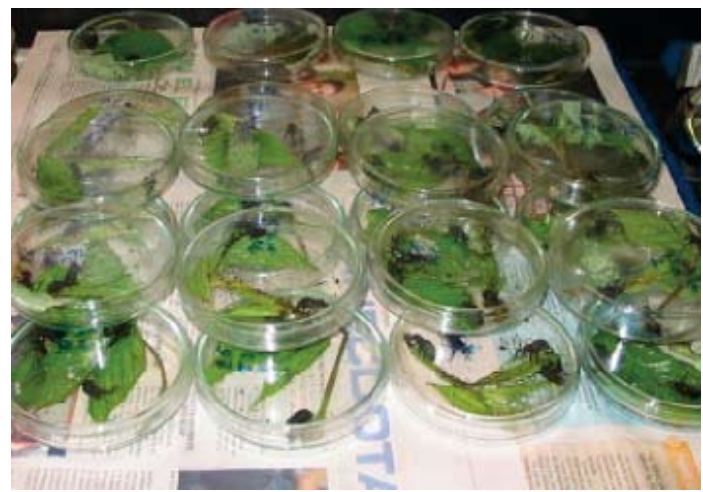

Figura 3. Ensayo con azadirachtina. 
bajo estas condiciones hasta la eclosión. Luego de la eclosión de los huevos se contabilizó la cantidad de larvas emergidas en cada unidad experimental. Paralelamente al periodo establecido para el ensayo se continuo el registro de oviposición diario hasta la octava semana.

El diseño experimental utilizado en este ensayo fue el de un experimento univariado, con cinco tratamientos (dosis + testigo) y cuatro repeticiones, dispuestos en bloques completamente al azar, donde el factor de estudio fue el efecto de la azadirachtina (Neem) en la embriogénesis del CF y las variables de respuestas fueron número de huevos y número de larvas eclosionadas. Los análisis estadísticos utilizados fueron el de varianza, prueba de rango múltiple de Duncan al 5\% de probabilidad, grado de efectividad o eficacia según Abbott (1925 citado por Unterstenhoefer, 1963) para comparación entre los promedios de los tratamientos y Probit utilizando las tablas de Fisher \& Yates (1963).

\section{RESULTADOS Y DISCUSIÓN}

Los resultados de este experimento se presentan en los Cuadros 1, 2, 3 y las Figuras 4, 5, 6.

En el Cuadro 1 se presentan los valores promedio de oviposición del CF durante un periodo de 30 días por cada tratamiento. El tratamiento con mayor postura correspondió al testigo (T0) sin aplicación de Neem con 174,75 huevos, también este tratamiento presentó la mayor desviación estándar correspondiente a $69,11 \mathrm{y}$ el mayor rango con una diferencia de 166 huevos. La menor variación se obtuvo en el tratamiento con $1 \mathrm{ml}$ de Neem por litro de agua (T2) con una diferencia entre sus rangos de 26 huevos.

La menor postura se registró en el tratamiento con $2 \mathrm{ml}$ de Neem por litro de agua (T3) con 36,5 huevos. Sin embargo, este tratamiento no tuvo diferencias significativas de postura respecto a las otras dosis utilizadas en el ensayo. En cambio todas las dosis utilizadas fueron significativas con respecto al testigo sin aplicación de Neem.

La postura semanal acumulada durante ocho semanas se puede observar en la Figura 3, donde se aprecia que hasta la séptima semana la mayor postura se obtuvo en el tratamiento testigo sin aplicación de Neem (T0), concentrándose mayoritariamente entre la primera y cuarta semana, manteniéndose en un ritmo relativamente estable entre la cuarta y octava semana.

En cuanto al número total de huevos por tratamiento, durante la séptima semana el tratamiento testigo sin aplicación de Neem (T0) continuaba presentando una mayor postura con respecto a los tratamientos con Neem. Durante la octava semana la postura acumulada del tratamiento con $4 \mathrm{ml} \mathrm{de}$ Neem por litro de agua (T4) y del tratamiento con $5 \mathrm{ml}$ por litro de agua (T5) superaron la postura acumulada del tratamiento testigo sin aplicación de Neem (T0), en cambio el tratamiento con $2 \mathrm{ml}$ de Neem por litro de agua (T3), en la octava semana, no registró postura, hecho que también ocurrió en la cuarta semana.

Entre los tratamientos con Neem, el correspondiente a $0,5 \mathrm{ml}$ de Neem por litro de agua (T1) siempre presentó una postura semanal acumulada mayor que el resto de los tratamientos con Neem. Además se puede observar que el comportamiento

\section{Cuadro 1}

Ovipostura durante 30 días

\begin{tabular}{|l|c|c|c|}
\hline \multicolumn{1}{|c|}{ Tratamientos } & $\mathbf{N}^{\mathbf{0}}$ de huevos & Desviación estándar & Rango \\
\hline (T0) Testigo & $174,75 \mathrm{a}$ & 69,11 & $94-260$ \\
\hline (T1) Neem $0,5 \mathrm{ml} / 1$ & $92,25 \mathrm{~b}$ & 53,46 & $50-184$ \\
\hline (T2) Neem $1 \mathrm{ml} / 1$ & $58,25 \mathrm{~b}$ & 9,44 & $44-70$ \\
\hline (T3) Neem $2 \mathrm{ml} / 1$ & $36,5 \mathrm{~b}$ & 13,57 & $28-60$ \\
\hline (T4) Neem $4 \mathrm{ml} / 1$ & $50,25 \mathrm{~b}$ & 19,25 & $19-70$ \\
\hline (T5) Neem $5 \mathrm{ml} / 1$ & $50,75 \mathrm{~b}$ & 39,35 & $15-117$ \\
\hline
\end{tabular}




\section{Cuadro 2}

Número de larvas emergidas durante 30 días

\begin{tabular}{|l|c|c|c|}
\hline \multicolumn{1}{|c|}{ Tratamientos } & Larvas emergidas & Desviación estándar & Rango \\
\hline (T0) Testigo & $111,0 \mathrm{a}$ & 48,37 & $43-166$ \\
\hline (T1) Neem 0,5 ml/1 & $45,5 \mathrm{~b}$ & 30,79 & $15-96$ \\
\hline (T2) Neem $1 \mathrm{ml} / 1$ & $24,25 \mathrm{~b}$ & 6,38 & $17-97$ \\
\hline (T3) Neem $2 \mathrm{ml} / \mathrm{l}$ & $16,25 \mathrm{~b}$ & 1,79 & $14-19$ \\
\hline (T4) Neem 4 ml/1 & $22,5 \mathrm{~b}$ & 12,89 & $2-34$ \\
\hline (T5) Neem $5 \mathrm{ml} / 1$ & $14,25 \mathrm{~b}$ & 12,48 & $4-57$ \\
\hline
\end{tabular}

Cuadro 3

Porcentaje de emergencia de larvas

\begin{tabular}{|l|c|c|c|}
\hline \multicolumn{1}{|c|}{ Tratamientos } & Emergencia (\%) & Desviación estándar & Rango \\
\hline (T0) Testigo & $62,38 \mathrm{a}$ & 12,02 & $45,74-73,55$ \\
\hline (T1) Neem 0,5 ml/1 & $46,72 \mathrm{ab}$ & 11,95 & $30,0-62,31$ \\
\hline (T2) Neem 1ml/1 & $42,06 \mathrm{ab}$ & 10,04 & $27,14-51,6$ \\
\hline (T3) Neem 2 ml/1 & $48,6 \mathrm{ab}$ & 14,97 & $31,66-57,14$ \\
\hline (T4) Neem 4 ml/1 & $38,73 \mathrm{~b}$ & 16,87 & $10,52-54,09$ \\
\hline (T5) Neem 5 ml/1 & $29,08 \mathrm{~b}$ & 12,2 & $9,75-43,33$ \\
\hline
\end{tabular}

de postura acumulada semanal entre el tratamiento con $4 \mathrm{ml}$ de Neem por litro de agua (T4) y el tratamiento con $5 \mathrm{ml}$ de Neem por litro de agua (T5) fue muy similar, situándose en una posición intermedia el tratamiento con $1 \mathrm{ml}$ de Neem por litro de agua (T2).

En el Cuadro 2 se presenta el promedio de larvas emergidas por tratamiento durante los 30 días de registro, observándose que el tratamiento con mayor eclosión de huevos fue el testigo sin aplicación de Neem (T0) con 111 larvas. La menor eclosión se registró en el tratamiento con $5 \mathrm{ml}$ de Neem por litro de agua (T5) con 14,25 larvas. El testigo sin aplicación de Neem (T0) tuvo diferencias significativas con el resto de los tratamientos. Sin embargo, respecto a la cantidad de larvas emergidas entre los tratamientos con Neem no hubo diferencias significativas entre ellos.

Según lo expuesto en el Cuadro 2 el testigo sin aplicación de Neem (T0) mostró la mayor desviación estándar con un 48,37 y una diferencia entre los rangos correspondiente a 123 larvas emergidas. La menor variación se obtuvo en el tratamiento con dos cc de Neem por litro de agua (T3) cuya diferencia de rango correspondió a cinco larvas emergidas.

La emergencia semanal acumulada de larvas del CF durante nueve semanas se puede observar en la Figura 4.

En la Figura 4 se aprecia que la mayor eclosión durante el periodo de ensayo correspondió al tratamiento sin aplicación de Neem (T0), lo que es coincidente con la mayor postura lograda en el tratamiento testigo sin aplicación de Neem (T0). La menor eclosión durante el periodo de ensayo correspondió al tratamiento con $5 \mathrm{ml}$ de Neem por litro de agua (T5), logrando un repunte en la octava semana. Además se puede observar que en el tratamiento con $0,5 \mathrm{ml}$ de Neem por litro de agua (T1) la emergencia de larva ocupó una posición intermedia entre los tratamientos con Neem. En la novena semana la emergencia de larvas fue mínima para la mayoría de los tratamientos. 


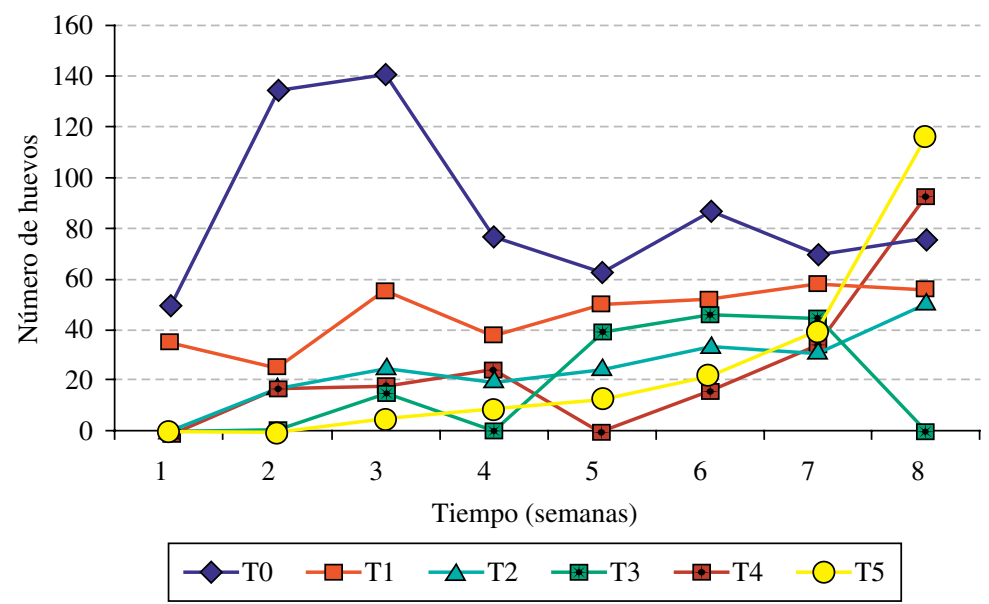

Figura 4. Postura semanal acumulada del CF por tratamiento.

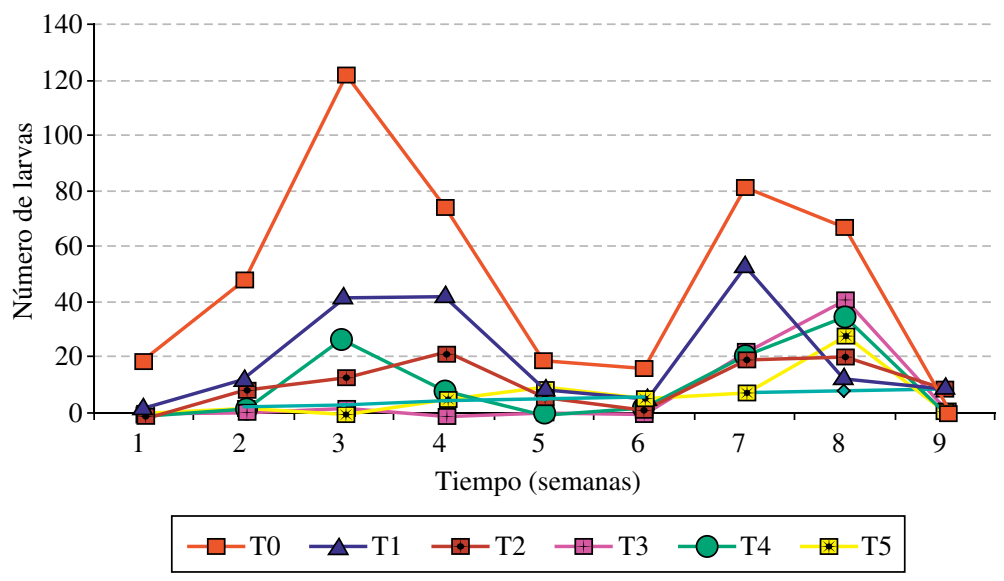

Figura 5. Emergencia semanal acumulada de larvas del CF.

En el Cuadro 3 se presenta el porcentaje promedio de larvas emergidas en el ensayo, obteniéndose en el tratamiento con $4 \mathrm{ml}$ de Neem por litro de agua (T4) y el tratamiento con $5 \mathrm{ml}$ de Neem por litro de agua (T5) diferencias significativas con respecto al testigo sin aplicación de Neem (T0); en este último la emergencia de larvas fue de un $62,38 \%$ y en los tratamientos mencionados los porcentajes de emergencia tuvieron una diferencia significativa con respecto al testigo de 54,34\%. Sin embargo, el tratamiento con $4 \mathrm{ml}$ de Neem por litro de agua (T4) y en el tratamiento con $5 \mathrm{ml}$ de Neem por litro de agua (T5) no presentaron diferencias significativas con respecto al resto de los tratamientos con Neem. El testigo sin aplicación de Neem (T0) a su vez no presentó diferencia significativa con respecto a los tratamientos con $0,5 \mathrm{ml}$ de Neem por litro de agua (T1), tratamiento con $1 \mathrm{ml}$ de Neem por litro de agua (T2) y tratamiento con $2 \mathrm{ml}$ de Neem por litro de agua (T3).

La mayor desviación estándar con respecto al promedio se produjo en el tratamiento con $4 \mathrm{ml}$ de Neem por litro de agua (T4); la menor variación y el menor rango se obtuvo en el tratamiento con 0,5 $\mathrm{ml}$ de Neem por litro de agua (T1) y el rango con mayor diferencia se presentó en el tratamiento con $4 \mathrm{ml}$ de Neem por litro de agua (T4).

Considerando el grado de eficacia de los tratamientos del Cuadro 4, se aprecia que todos los tratamientos con Neem manifestaron diferencias 
significativas con respecto al testigo sin aplicación de Neem (T0). El tratamiento con $5 \mathrm{ml}$ de Neem por litro de agua (T5) fue significativamente diferente en cuanto a su efectividad respecto a las dosis de los tratamientos con $0,5 \mathrm{ml}$ de Neem por litro de agua (T1), tratamiento con $1 \mathrm{ml}$ de Neem por litro de agua (T2), tratamiento con $4 \mathrm{ml}$ de Neem por litro de agua (T4), pero con respecto al tratamiento con $2 \mathrm{ml}$ de Neem por litro de agua (T3) no presentó diferencias significativas.

La Figura 6 representa la determinación de la dosis media efectiva (DE50) y la DE90 en función de los grados de eficacia de las diferentes dosis de Neem aplicadas en este ensayo. En efecto, la DE50 calculada corresponde a $0,0290 \mathrm{ml}$ de Neem por litro de agua y la DE90 se determinó en $8,923 \mathrm{ml}$ de Neem por litro de agua.

En su trabajo Schmutterer (1987) concluye que el Neem tiene efecto sobre la fecundidad de las hembras del escarabajo colorado de la papa, mani- festándose en una baja postura del insecto, lo que concuerda con lo ocurrido con el CF (Cuadro 1). El mismo autor en sus ensayos destaca que el alimento tratado con Neem ejerció una fuerte acción antialimentaria; en el CF este aspecto no fue evaluado, sí la emergencia de larvas de aquellas hembras del CF que consumieron material vegetal tratado con el extracto vegetal (Cuadro 2), resultando una disminución significativa en el porcentaje de emergencia como ocurrió también en los ensayos sobre el escarabajo colorado de la papa llevados a cabo por el autor anteriormente citado.

Respecto al efecto de la dosis de Neem, Schmutterer (1987) comprobó que la disminución de la cantidad de huevos puestos por las hembras disminuyó en la medida que se aumentó la dosis. Este efecto concuerda con la cantidad de huevos dejados por las hembras del CF, según la dosis utilizada (Cuadro 2), excepto con las dosis de 4 y $5 \mathrm{ml}$ de Neem por litro de agua, que siendo signi-

\section{Cuadro 4}

Grado de efectividad $(\%)$ de los tratamientos

\begin{tabular}{|l|c|c|c|}
\hline \multicolumn{1}{|c|}{ Tratamientos } & Eficacia (\%) & Desviación estándar & Rango \\
\hline (T0) Testigo & $0,00 \mathrm{c}$ & 0 & 0 \\
\hline (T1) Neem 0,5 ml/1 & $70,69 \mathrm{~b}$ & 33,74 & $53,57-90,69$ \\
\hline (T2) Neem 1 ml/1 & $73,50 \mathrm{~b}$ & 11,43 & $60,46-86,98$ \\
\hline (T3) Neem 2 ml/1 & $79,64 \mathrm{ab}$ & 14,17 & $55,81-90,40$ \\
\hline (T4) Neem 4 ml/1 & $72,26 \mathrm{~b}$ & 17,89 & $51,16-98,7$ \\
\hline (T5) Neem 5 ml/1 & $86,27 \mathrm{a}$ & 7,68 & $76,02-96,98$ \\
\hline
\end{tabular}

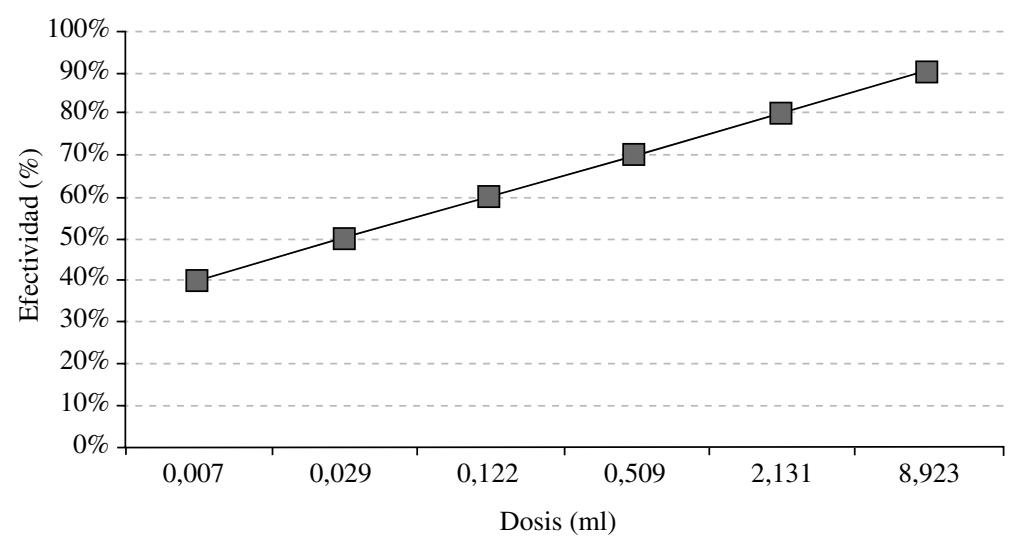

Figura 6. DE50 y DE90 según el grado de eficacia. 
ficativamente diferente al testigo sin aplicación de Neem, no lo fue respecto a las dosis de $0,5,1$ y 2 $\mathrm{ml}$ de Neem por litro de agua, pero en cuanto a la emergencia de larvas ésta fue disminuyendo en la medida que se aumentó la dosis.

\section{CONCLUSIONES}

La azadirachtina, formulada como Neem, tiene un efecto en la inhibición de la embriogénesis en Aegorhinus superciliosus (Guérin) (Coleoptera: Curculionidae).

Las diferencias significativas de Neem con respecto al testigo sin aplicación (T0) se manifestaron tanto en la ovipostura, emergencia de larvas, porcentaje de emergencia y también en el grado de eficacia.

El aumentar las dosis de Neem fue disminuyendo progresivamente la postura del CF; lo mismo ocurrió respecto a la emergencia de larvas del CF, contrastar los resultados con un testigo sin aplicación del Neem. La menor emergencia después de 30 días se registró en el tratamiento con $5 \mathrm{ml}$ de Neem por litro de agua (T5) con un promedio de 29,08 larvas.

En general, al aumentar las dosis de Neem se consigue un mayor grado de eficacia en la reducción de la embriogénesis del CF. El tratamiento con $5 \mathrm{ml}$ de Neem por litro de agua (T5) presentó el mayor porcentaje de efectividad con un $86,27 \%$.

La dosis efectiva media (DE50) fue de $0,0290 \mathrm{ml}$ de Neem por litro de agua y la dosis efectiva 90 (DE90) fue de $8,923 \mathrm{ml}$ de Neem por litro de agua.

\section{LITERATURA CITADA}

AGUILERA, A. 1994. Insectos y ácaros en frambuesa en la IX Región. Investigación y Progreso Agropecuario, INIA Carillanca (Temuco, Chile) 12 (4): 27.

AGUILERA, A. 1995. Control selectivo de plagas en frutales en la zona sur. Seminario de protección vegetal INIA Carillanca (Temuco, Chile) 45: 141-188.

AGUILERA, A. \& REBOLLEDO, R. 2001. Estados larvarios de Aegorhinus superciliosus (G.) (Coleoptera: Curculionidae). Rev. Chilena de Entomol. 28: 5-8.

ASCHER, K.R.S. 1981. Some physical (solubility) properties and biological (sterilant for Epilachna varivestis females) effects of a dried methanolic neem (Azadirachta indica) seed kernel extract. Proc. $1^{\text {st }}$ Int. Neem Conf. pp. 63-74.

ASCHER, K.R.S.; ELIYAHU, M.; NEMNY, N.E. \& MEISNER, J. 1984. Neem seed kernel extract as an inhibitor of growth and fecundity in Spodoptera litoralis. Proc. $2^{\text {nd }}$ Int. Neem Conf. pp. 331-334.

ASCHER, K.R.S.; NEMNY, N.E.; BLUMBERG, D. \& GOLDENBERG, S. 1986. Egg sterilizing effect of benzoylphenylureas via the adult stage of the nitidulid beetle, Carpophilus hemiptessrus. Phytoparasitica 14: 187-192.

DEVOTTO, L. \& GERDING, M. 2001. Plagas de los berries en la zona centro sur. Tierra Adentro 36: 12-14.

FISHER, R. \& YATES, F. 1963. Statistical tables for biological agricultural and medical research. Sixth Edition Hafner Publishing Company Inc. (New York). 146 p.

GERDING G., M.; FRANCE, A.; GERDING P., M. \&. CISTERNAS, E. 2002. Control de plagas con hongos entomopatógenos. Tierra Adentro (Chile) 43: 45-47.

GONZÁLEZ, R. 1985. Plagas de importancia cuarentenaria en la frambuesa de exportación. Revista Frutícola (Chile) 6 (3): 75-82.

GONZÁLEZ, R. \& VARAS, P. 1989. Manejo de plagas de la frambuesa. Degradación de residuos del piretroide fluvalinato. Revista Frutícola (Chile) 10 (2): 64-70.

GUERRERO, J. \& AGUILERA, A. 1989. Plagas y enfermedades del arándano chileno (Vacciun corimbosum L.). Próxima Década (Santiago, Chile)7 (76):24-29.

GUERRERO, J.; CARRILLO, R. \& AGUILERA, A. 1999. Caracterización morfológica y germinación de cepas del hongo entomopatógeno Metarhizium anisopliae, var. anisopliae, asociado a larvas de escarabaeidos y curculiónido. Agro Sur (Valdivia, Chile) 27 (2): 23-24.

HEYDE, J. V.D.; SAXENA, R.C. \& SCHMUTTERER, H. 1984. Neem oil and neem extracts as potencial insecticides for control of hemipterous rice pest. Proc. $2^{\text {nd }}$ Int. Neem Conf. pp. 377-390.

NEIRA, A. \& MUNDACA, C. 1982. Detección de plagas de arbustos frutales en la Región de Los Lagos. Simiente (Santiago, Chile) 52 (3-4):121-125.

PRADO, E. 1991. Artrópodos y sus enemigos naturales asociados a plantas cultivadas en Chile. Instituto de Investigaciones Agropecuarias (Santigo, Chile). Boletín Técnico 169. 203 p.

REMBOLD, H. \& SIEBER, K.P. 1981. Inhibition of oogenesis and ovarian ecdysteroid synthesis by azadirachtin in Locusta migratoria migratorioides (R. \& F.). Z. Naturforsch. 36c: 466-469.

RODRÍGUEZ, M.; FRANCE, A. \& GERDING, M. 2005. Control biológico de plagas del suelo. Tierra Adentro (Chile) 62: 12-13.

SCHMIDT, G.H. \& PESEL, E. 1986. Studies of the sterilizing effect of Neem extracts in ants. Proc. $3^{\text {rd }}$ Neem Conf. pp. 361-373.

SCHMUTTERER, H. 1987. Fecundity reducing and sterilizing effects of Neem seed kernel extracts in the Colorado potato beetle, Leptinotarsa decemlineata. Proc. $3^{\text {rd }}$ Int. Neem Conf. pp. 351-360.

SCHULZ, W.D. 1981. Pathological alterations in the ovarios of Epilachna varivestis induced by an extract from Neem kernels. Proc. $1^{\text {st }}$ Int. Neem Conf. pp. 81-96.

SOLER, A. 1994. Efectividad de algunos reguladores del crecimiento en la inhibición de la embriogénesis de Aegorhinus superciliosus (G.) (Coleoptera: Curculionidae).Tesis Ingeniero Agrónomo Facultad de Ciencias Agropecuarias, Universidad de La Frontera (Temuco, Chile). 189 p. 
STEETS, R. 1976. Zur Wirkung eines gereinigten Extraktes aus Früchten von Azadirachta indica A Juss auf Leptinotarsa decemlineata Say (Coleoptera: Chrysomelidae). Z. Angew. Ent. 82:169-176.

STEETS, R. \& SCHMUTTERER, H. 1975. Der Einfufb von Azadirachtin auf die Lebensdauer und das
Reproduktionsvermögen von Epilachna varivestis Muls. (Coleoptera: Coccinellidae). Z. Pflkrankh. PflSchutz 82: 176-179.

UNTERSTENHOEFER, G. 1963. Las bases para ensayos fitosanitarios de campo. Pflanzenschutz Nachrichten "Bayer" 16 (3): 89-175. 
